\title{
Mamíferos (Mammalia) de San José del Guaviare, Colombia
}

\section{Mammals (Mammalia) of San José del Guaviare, Colombia}

\author{
Hugo Fernando López Arévalo ${ }^{\circ} \square$, Darwin Manuel Morales-Martínez ${ }^{\square} \square$, \\ Catherine Mora-Beltrán ${ }^{\mathbb{}} \square$, María C. Calderón-Capote ${ }^{\mathbb{}} \square$, Catalina Cárdenas-González ${ }^{\circ} \square$, \\ Natalia Atuesta-Dimian $\square$, Marco J. Melo ${ }^{\circ} \square$, Wilmer Ramírez ${ }^{\circ} \square$
}

\section{Resumen}

Presentamos los resultados obtenidos durante cuatro años de muestreo de mamíferos en diferentes coberturas vegetales del municipio de San José del Guaviare, Guaviare, Colombia. Entre los años 2012 y 2017 recolectamos información de la diversidad de mamíferos a través del muestreo con redes de niebla, trampas para pequeños y medianos mamíferos, cámaras trampa, observaciones directas, entrevistas e información secundaria. Reportamos 121 especies de mamíferos nativos para el municipio. Registramos una completitud del muestreo de $64 \%$ para murciélagos, $60 \%$ para pequeños mamíferos no voladores y de $100 \%$ para medianos y grandes mamíferos. Destacamos dos novedades geográficas para los marsupiales Marmosa waterhousei y Glironia venusta, y seis novedades en los murciélagos Lonchorhina marinkellei, Phyllostomus latifolius, Glyphonycteris sylvestris, Glyphonycteris daviesi, Glossophaga commissarisi y Myotis keaysi. La riqueza encontrada es comparable con otros inventarios a largo plazo de mamíferos neotropicales, y presenta la mayor diversidad de especies de murciélagos y primates reportados en Colombia. Sin embargo, es necesario seguir realizando muestreos intensivos, ya que la riqueza de grupos como pequeños mamíferos no voladores y murciélagos está aún subestimada.

Palabras clave. Amazonía. Diversidad. Guayana. Inventario. Murciélagos. Primates. Riqueza.

\begin{abstract}
We present the results obtained during four years of sampling of mammals in different plant covers of the municipality of San José del Guaviare, Guaviare, Colombia. Between 2012 and 2017 we collected information about diversity of mammals, through capture with mist nets, traps for small and medium mammals, camera traps, direct observations, interviews, and secondary information. We report 121 species of mammals for the municipality. We recorded sampling completeness of $64 \%$ for bats, $60 \%$ for small non-flying mammals, and 100 $\%$ for medium and large mammals. We highlight geographical novelties for the marsupials Marmosa waterhousei, Glironia venusta and for the bats Lonchorhina marinkellei, Phyllostomus latifolius, Glyphonycteris sylvestris, Glyphonycteris daviesi, Glossophaga commissarisi and Myotis keaysi. The richness found in this study is comparable with other long-term neotropical inventories and the area has the largest number of bat and primate species reported for any site in Colombia. However, it is necessary to continue with the intensive sampling, because the richness of small non-volant mammals and bats are still underestimated.
\end{abstract}

Keywords. Amazon. Bats. Diversity. Guiana. Inventory. Primates. Richness. 


\section{Introducción}

A diferencia de algunas localidades de Centro y Suramérica, en Colombia es escasa la información publicada de muestreos sistemáticos de más de dos años en los que se preserven series de especímenes de referencia (Voss \& Emmons, 1996; Simmons \& Voss, 1998; Voss et al., 2001; Lim \& Engstrom 2005; Solari et al., 2006). Este vacío de información ocasiona que las localidades colombianas no se incluyan en análisis regionales sobre la diversidad de mamíferos en bosques neotropicales (e.g.Voss \& Emmons 1996), y explica también la escasez de poblaciones de mamíferos colombianos en estudios filogenéticos, dada la baja disponibilidad de tejidos y secuencias genéticas (e.g. Redondo et al., 2008). En consecuencia, se tiene un conocimiento fragmentado de la biodiversidad colombiana, el cual es fundamental para la evaluación y planificación de estrategias de conservación y la resolución de hipótesis de distribución de la diversidad y de biogeografía en el país.

Durante los últimos años ha sido posible el acceso a algunas regiones que habían permanecido aisladas por el conflicto armado, como es el caso del departamento de Guaviare. Este departamento se ubica en una zona estratégica para la biodiversidad, debido a la confluencia de distintas provincias biogeográficas como la Amazonia, la Orinoquia, y la Guayana (Hernández-Camacho et al., 1992). Aunque el municipio de San José del Guaviare (departamento del Guaviare) cuenta con recientes inventarios cortos de mamíferos realizados por investigadores invitados del Field Museum of Natural History (Montenegro \& Restrepo, 2018, reportando 48 especies), y la Fundación para la Conservación y el Desarrollo Sostenible (Agudelo-Liz et al., 2018, reportando 50 especies), además de guías de divulgación que buscan brindar información sobre los mamíferos, aplicable en actividades turísticas (e.g. López-Arévalo et al., 2019), no existe un compendio que reúna la diversidad de la mastofauna del municipio o del departamento.

Nuestro inventario incluye los resultados de cuatro años de muestreo en el municipio de San José del Guaviare, Guaviare, Colombia. El objetivo del presente estudio es presentar una lista de mamíferos para el municipio basado en evidencia de salidas de campo, registros de colecciones y revisión de literatura. Adicionalmente, estimamos la riqueza de especies, la abundancia y la representatividad del muestreo para algunos grupos de mamíferos en esta zona.

\section{Materiales y métodos}

\section{Área de estudio}

El municipio de San José del Guaviare ocupa la parte norte del departamento del Guaviare, con una extensión de $42327 \mathrm{~km}^{2}$. Presenta un complejo panorama biológico debido a que confluyen allí diferentes provincias biogeográficas y elementos florísticos de la Amazonia, la Orinoquia y la Guayana (Hernández Camacho et al., 1992; Barona-Colmenares et al., 2018). La zona presenta un régimen climático bimodal, con una precipitación promedio anual de $2628 \mathrm{~mm}$ y una temperatura promedio de $25.7^{\circ} \mathrm{C}$. La época seca se extiende desde diciembre hasta marzo, con un mínimo de lluvias en enero $(30 \mathrm{~mm})$ y un periodo de lluvias desde abril hasta noviembre, con un máximo en junio $(560 \mathrm{~mm})$. El muestreo comprendió 18 localidades que corresponden a varios tipos de coberturas vegetales, incluyendo bosques secundarios de galería o de planicie aluvial en cercanía al río Guaviare; bosques de tierra firme adyacentes a afloramientos rocosos, de filiación Amazónica; y herbazales y arbustales sobre afloramientos rocosos del escudo Guayanés (Figura 1; Tabla 1). La descripción de estas coberturas en San José del Guaviare se presenta en Barona-Colmenares et al. (2018).

Recolección de datos. El inventario incluye los resultados de seis muestreos independientes entre 2012 y 2017, cinco de ellos realizados entre 2012 y 2014 por estudiantes y docentes del Programa de Biología de la Universidad Nacional de Colombia, en la asignatura Taxonomía animal, y un inventario realizado por el Instituto Amazónico de Investigaciones Científicas SINCHI en el año 2017. Se muestrearon murciélagos, pequeños mamíferos no voladores y mamíferos medianos y grandes, estos clasificados según las categorías de tamaño presentadas en Sánchez et al. (2004).

Para la captura de murciélagos se utilizaron entre tres y 15 redes de niebla por noche de 6 a 12 metros de longitud cada una, ubicadas entre $0.5 \mathrm{~m}$ y $3 \mathrm{~m}$ de altura desde el suelo, durante un periodo comprendido entre las 18:00 h y las 00:00 h. Calculamos el esfuerzo de muestreo como el número de metros lineales de red por hora (metros-hora-red). Reportamos un esfuerzo total de 10666 metros-hora-red para todo el inventario. Adicionalmente se realizaron muestreos complementarios en cuevas utilizando redes de niebla, captura manual y redes entomológicas manuales. 


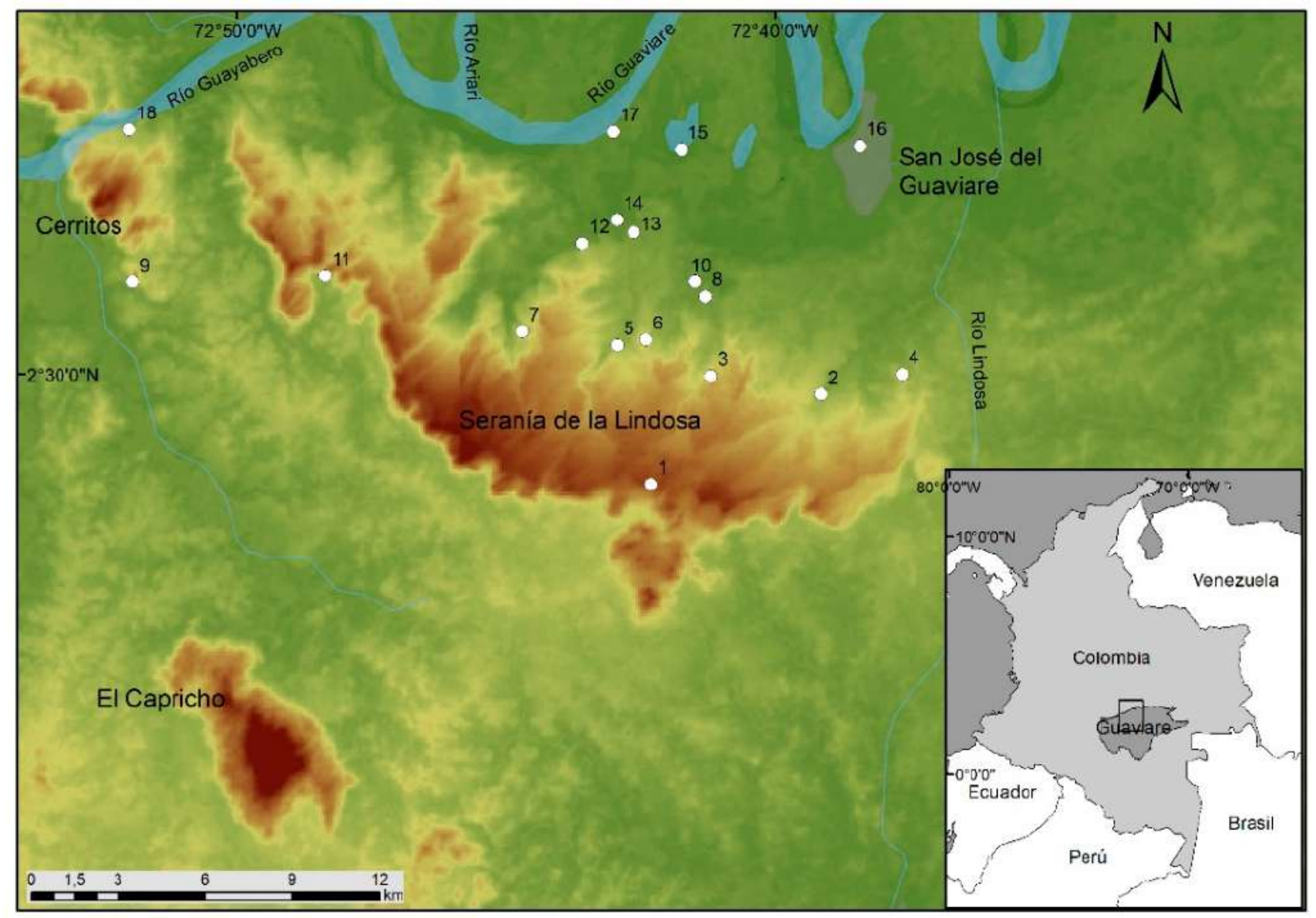

Figura 1. Ubicación geográfica de las 18 localidades muestreadas en el inventario de mamíferos de San José del Guaviare, Colombia. Los nombres y las coordenadas de cada sitio se muestran en la Tabla 1.

Figure 1. Geographic location of 18 localities sampled in the inventory of mammals of San José del Guaviare, Colombia. The names and coordinates are shown in Table 1.

La captura de pequeños mamíferos no voladores (PMNV) y medianos mamíferos fue realizada mediante el uso de trampas Sherman y trampas Tomahawk en tres localidades: El Retiro, Isla Laguna Negra, y Finca Puerto Amor (Playa Güio) (localidades 12, 15 y 17) entre los años 2012 y 2014. Se utilizaron de 40 a 50 trampas Sherman y de una a tres trampas Tomahawk por salida, las cuales se ubicaron por estaciones en transectos lineales con una separación de por lo menos 10 metros entre trampas. Se calculó el esfuerzo de muestreo como el número de trampas por noche, para un esfuerzo de muestreo total de 8206 trampas-noche para las Sherman y de 134 trampas-noche para las Tomahawk.

Para mamíferos medianos y grandes se utilizaron de tres a cinco cámaras de detección automática (cámaras trampa) por salida entre los años 2012 y 2014, en las Veredas Playa Güio y El Retiro, y 18 cámaras trampa en el año 2017, en la Vereda El Raudal del Guayabero (localidades 12, 13, 17 y 18). Las cámaras fueron instaladas cerca de senderos de mamíferos, sitios de acceso al agua, o comederos. Las cámaras permanecieron activas de cinco a 12 noches y se configuraron para tomar fotografías y videos; se calculó el esfuerzo de muestreo como número de cámaras por noche para un total de 662 trampas-noche. Finalmente, para primates y mamíferos acuáticos se hicieron recorridos no sistemáticos de observación directa ad libitum, con el uso de binoculares y ocasionalmente se registraron otros mamíferos arborícolas y semiacuáticos. 
Tabla 1. Localidades de muestreo de mamíferos en el municipio de San José del Guaviare, Colombia, sus coordenadas geográficas, los tipos de cobertura vegetal principal, y los métodos utilizados en cada una.

Table 1. Localities sampled for mammals in San José del Guaviare, Colombia, their geographic coordinates, main plant cover, and the sampling methods used in each one.

\begin{tabular}{clcccc}
\hline Número & \multicolumn{1}{c}{ Localidades muestreadas } & Latitud (N) & Longitud (W) & $\begin{array}{c}\text { Tipos de } \\
\text { cobertura }\end{array}$ & $\begin{array}{c}\text { Métodos } \\
\text { empleados }\end{array}$ \\
\hline 1 & Ciudad de Piedra * & 2.46575 & -72.70511 & HB y AB & R, OO \\
\hline 2 & Pozos Naturales * & 2.49367 & -72.65231 & HB y AB & R, OO \\
\hline 3 & Los Túneles * & 2.49944 & -72.68656 & HB y AB & R, OO \\
\hline 4 & Diamante de las Aguas * & 2.50000 & -72.62722 & HB y AB & R, OO \\
\hline 5 & Tranquilandia * & 2.50889 & -72.71569 & HB y AB & R, OO \\
\hline 6 & Puerta de Orión * & 2.51064 & -72.70667 & HB y AB & R, OO \\
\hline 7 & Cascada Las Delicias * & 2.51311 & -72.74500 & HB y AB & R, OO \\
\hline 8 & Aqua Fresh * & 2.52408 & -72.68825 & HB y AB & R, OO \\
\hline 9 & Cerro Azul * & 2.52853 & -72.86589 & HB y AB & R, OO \\
\hline 10 & Embalse La María * & 2.52864 & -72.69139 & BTF & R, OO \\
\hline 11 & Los Alpes ** & 2.53047 & -72.80628 & BTF & R, OO \\
\hline 12 & El Retiro * & 2.54053 & -72.72631 & HB y AB & R, CT, T, OO \\
\hline 13 & Picapiedra * & 2.54400 & -72.71039 & HB y AB & R, CT, OO \\
\hline 14 & La Pradera * & 2.54787 & -72.71578 & BG & R, OO \\
\hline 15 & Isla Laguna Negra * & 2.56976 & -72.69578 & BG & R, T, OO \\
\hline 16 & San José del Guaviare * & 2.57083 & -72.64028 & BG & OO \\
\hline 17 & Finca Puerto Amor (Playa Güio) * & 2.57519 & -72.71694 & BG & R, CT, T, OO \\
\hline 18 & Angosturas II (Raudal del Guayabe- & 2.57586 & -72.86694 & BTF & R, CT, OO \\
\hline ro) * & & & & \\
\hline
\end{tabular}

Tipos de cobertura: BG, bosques secundarios de galería o de planicie aluvial, cerca del río Guaviare; BTF, bosques de tierra firme adyacentes a afloramientos rocosos; $\mathbf{H B}$ y $\mathbf{A B}$, herbazales y arbustales sobre afloramientos rocosos del escudo Guayanés. Métodos empleados: CT, cámara trampa; R, red de niebla; OO, observaciones ocasionales; T, trampas Sherman y Tomahawk. *Muestreada de 2012 a $2014 .{ }^{* *}$ Muestreada en 2017.

Todos los individuos capturados fueron identificados preliminarmente en campo, y en caso de no tener dudas con su identificación taxonómica fueron liberados. Se recolectó al menos un espécimen de cada morfotipo identificado en campo con el fin de confirmar su identificación taxonómica y realizar una colección de referencia de la zona. El material recolectado se preservó en seco (piel, cráneo y esqueleto) o en etanol al $70 \%$. Adicionalmente se recolectaron muestras de tejido de algunos ejemplares. Todo el material recolectado se depositó en la Colección de Mamíferos "Alberto Cadena García" del Instituto de Ciencias Naturales de la Universidad Nacional de Colombia (ICN 22303 al ICN 22675 e ICN 23829 al 23893).

La lista de especies obtenida en los muestreos en campo se complementó con información secundaria proveniente de entrevistas no estructuradas a pobladores 
mayores de 30 años que han vivido por lo menos diez años en la zona rural del municipio. Para facilitar las identificaciones se utilizaron guías visuales (Emmons \& Feer, 1997; Defler, 2003). A su vez, se incluyó una revisión de especímenes depositados en la Colección de Mamíferos "Alberto Cadena García" del Instituto de Ciencias Naturales, provenientes de la zona de estudio (Anexo 2), y una revisión bibliográfica de trabajos con mamíferos realizados en el departamento del Guaviare (Rodríguez-Castellanos et al., 2013; Jiménez-Ramírez, 2014; Agudelo-Liz et al. , 2018; Montenegro \& Restrepo, 2018). Para cada una de las especies se registró la categoría de amenaza reportada por la Unión Internacional para la Conservación de la Naturaleza (IUCN, 2020) y la resolución 1912 de Ministerio de Ambiente y Desarrollo Sostenible sobre el listado de las especies silvestres amenazadas de la diversidad biológica colombiana (Ministerio de Ambiente y Desarrollo Sostenible, 2017).

Análisis de datos. Estimamos la representatividad de nuestro inventario para las metodologías de redes de niebla, trampas Sherman y cámaras trampa a partir de curvas de rarefacción de especies y el modelo de Jackknife 2, que no asume homogeneidad en la muestra (Heltshe \& Forrester, 1983) y es menos sesgado cuando hay pocas especies representadas en una muestra (Gotelli \& Cowell, 2011). Para las redes de niebla y las trampas Sherman se consideró cada captura como una unidad de muestreo independiente y aleatoria (Willott, 2001). Para las cámaras trampa, la unidad de muestreo correspondió a un evento fotográfico independiente, donde los registros audiovisuales (fotografías o videos) de la misma especie fueron considerados si estaban separados por un intervalo de tiempo de mínimo una hora. Para evitar la influencia del orden de ocurrencia de las muestras realizamos 1000 aleatorizaciones y la completitud del inventario se estimó dividiendo el valor de riqueza obtenido por el valor de riqueza estimado por Jackknife 2 multiplicado por 100. No se calculó la completitud para la metodología de trampas Tomahawk debido al pequeño número de capturas obtenido por dicha metodología. Los análisis fueron hechos con el software EstimateS 9.1 (Colwell, 2013).

\section{Resultados}

Resumen del inventario. Reportamos 121 especies de mamíferos pertenecientes a 31 familias y diez órdenes (Anexo 1; Figuras 2 y 3). Además, registramos la presencia de los roedores invasores Rattus norvegicus y Rattus rattus. Mediante los métodos de muestreo utilizados se identificaron 111 especies, y con la información secundaria recopilada mediante métodos complementarios de registro se adicionaron diez especies (Anexo 1). El orden con mayor riqueza fue Chiroptera, con 63 especies, representando el $52 \%$ del total de las especies de mamíferos aquí reportadas. Del total de especies de murciélagos reportadas este estudio, 55 fueron registradas mediante muestreo en campo, y ocho mediante revisión de literatura y colecciones biológicas. El segundo orden con mayor riqueza fue Rodentia, con 13 especies, que representan el $11 \%$ de la riqueza; 12 especies fueron registradas en el muestreo y una por revisión de colecciones biológicas. El tercero fue el orden Carnivora, con 13 especies, que representan el $11 \%$ de la riqueza; diez de estas especies fueron registradas en el muestreo y tres en entrevistas y revisión de literatura. Finalmente, el orden Primates, con 11 especies registradas en el muestreo, representa el $9.4 \%$ de la riqueza (Anexo 1).

Mediante el uso de trampas Sherman se capturaron 14 individuos de ocho especies de PMNV: seis roedores y dos marsupiales. Con trampas Tomahawk solo se capturaron ejemplares de Didelphis marsupialis, Proechimys quadruplicatus y un individuo del género Proechimys que no pudo ser identificado a nivel de especie con la literatura actual. A partir de cámaras trampa se identificaron 17 especies de mamíferos medianos y grandes, correspondiendo la mayoría de los registros a las especies D. marsupialis, Cuniculus paca y Dasyprocta fuliginosa. Se destacan registros de Procyon cancrivorus, Nasua nasua, Tapirus terrestris y Eira barbara. Por observación se identificaron 11 especies de primates (Anexo 1); entre ellas, las especies más abundantes incluyen a Alouatta seniculus con grupos de tres a seis individuos incluyendo crías; Lagothrix lagotricha, con grupos de tres a diez individuos con crías; Saimiri cassiquiarensis con los grupos más grandes, en los que se contabilizaron hasta 20 individuos; con menor frecuencia se observaron Aotus vociferans con dos individuos, y finalmente para las especies Ateles belzebuth y Cacajao melanocephalus se reportó un individuo. Otras especies de primates observadas ocasionalmente incluyen Sapajus apella, Cheracebus lugens, Saguinus inustus y Plecturocebus ornatus. Otros mamíferos observados durante el periodo del muestreo incluyen Bradypus variegatus, Caluromys lanatus, Lontra longicaudis, Microsciurus flaviventer e Inia geoffrensis.

En entrevistas hechas a ocho pobladores de las veredas Playa Güio, El Retiro y La Lindosa se reconocieron 31 especies de mamíferos pertenecientes a nueve órdenes; de estas se reportan diez especies que no se registraron por otra metodología en campo, incluyendo felinos 

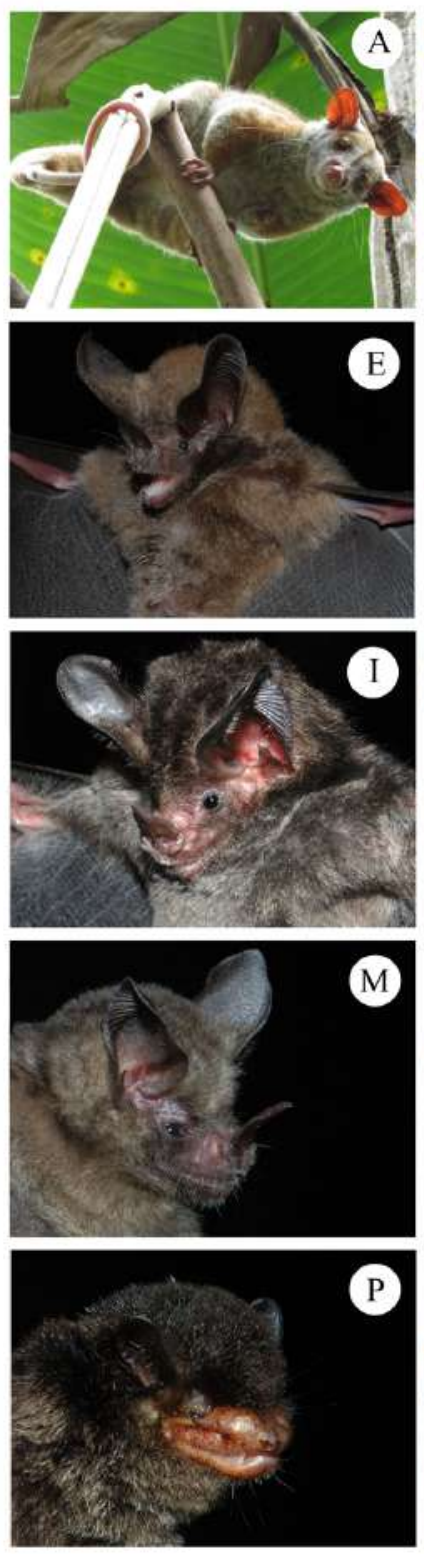
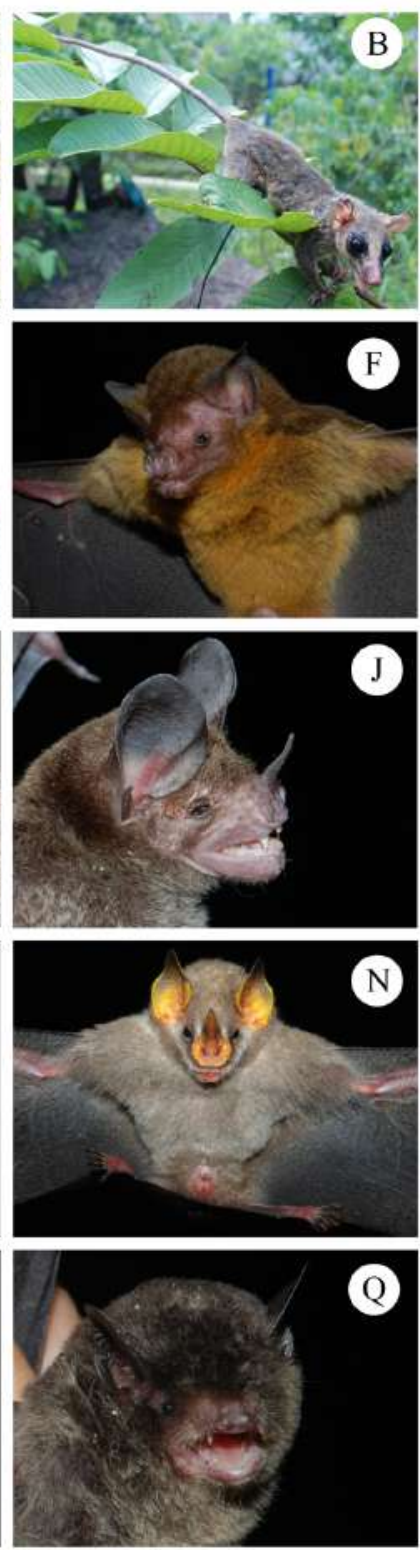
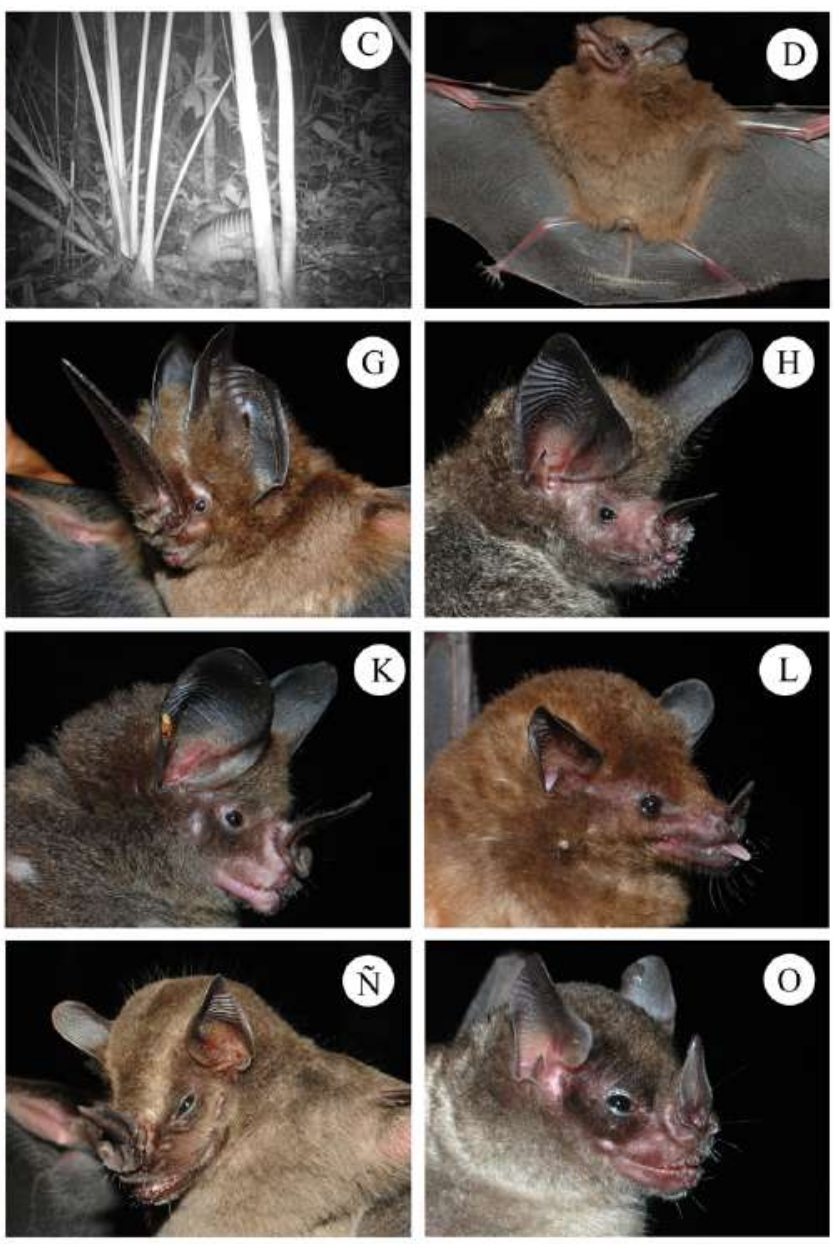

Figura 2. Algunas especies de mamíferos registrados en el municipio de San José del Guaviare, Colombia. A, Caluromys lanatus; B, Marmosa waterhousei; C, Dasypus novemcinctus; D, Peropteryx macrotis; E, Micronycteris megalotis; F, Lampronycteris brachyotis; G, Lonchorhina marinkellei; H, Trachops cirrhosus; I, Tonatia maresi; J, Phylloderma stenops; K, Phyllostomus latifolius; L, Hsunycteris thomasi; M, Glyphonycteris sylvestris; N, Mesophylla macconnelli; N, Platyrrhinus infuscus; O, Sturnira tildae; P, Eptesicus chiriquinus; Q, Myotis keaysi. Fotografías: B, D-Q, Instituto SINCHI; C, Grupo en Conservación y Manejo de Vida Silvestre, Universidad Nacional de Colombia Universidad Nacional de Colombia; A, Wilmer A. Ramírez.

Figure 2. Some mammal especies recorded in the municipality of San José del Guaviare. A, Caluromys lanatus; B, Marmosa waterhousei; C, Dasypus novemcinctus; D, Peropteryx macrotis; E, Micronycteris megalotis; F, Lampronycteris brachyotis; G, Lonchorhina marinkellei; H, Trachops cirrhosus; I, Tonatia maresi; J, Phylloderma stenops; K, Phyllostomus latifolius; L, Hsunycteris thomasi; M, Glyphonycteris sylvestris; N, Mesophylla macconnelli; Ñ, Platyrrhinus infuscus; O, Sturnira tildae; P, Eptesicus chiriquinus. Photographs: B, D-Q, Instituto SINCHI; C, Grupo en Conservación y Manejo de Vida Silvestre, Universidad Nacional de Colombia Universidad Nacional de Colombia; A, Wilmer A. Ramírez. 

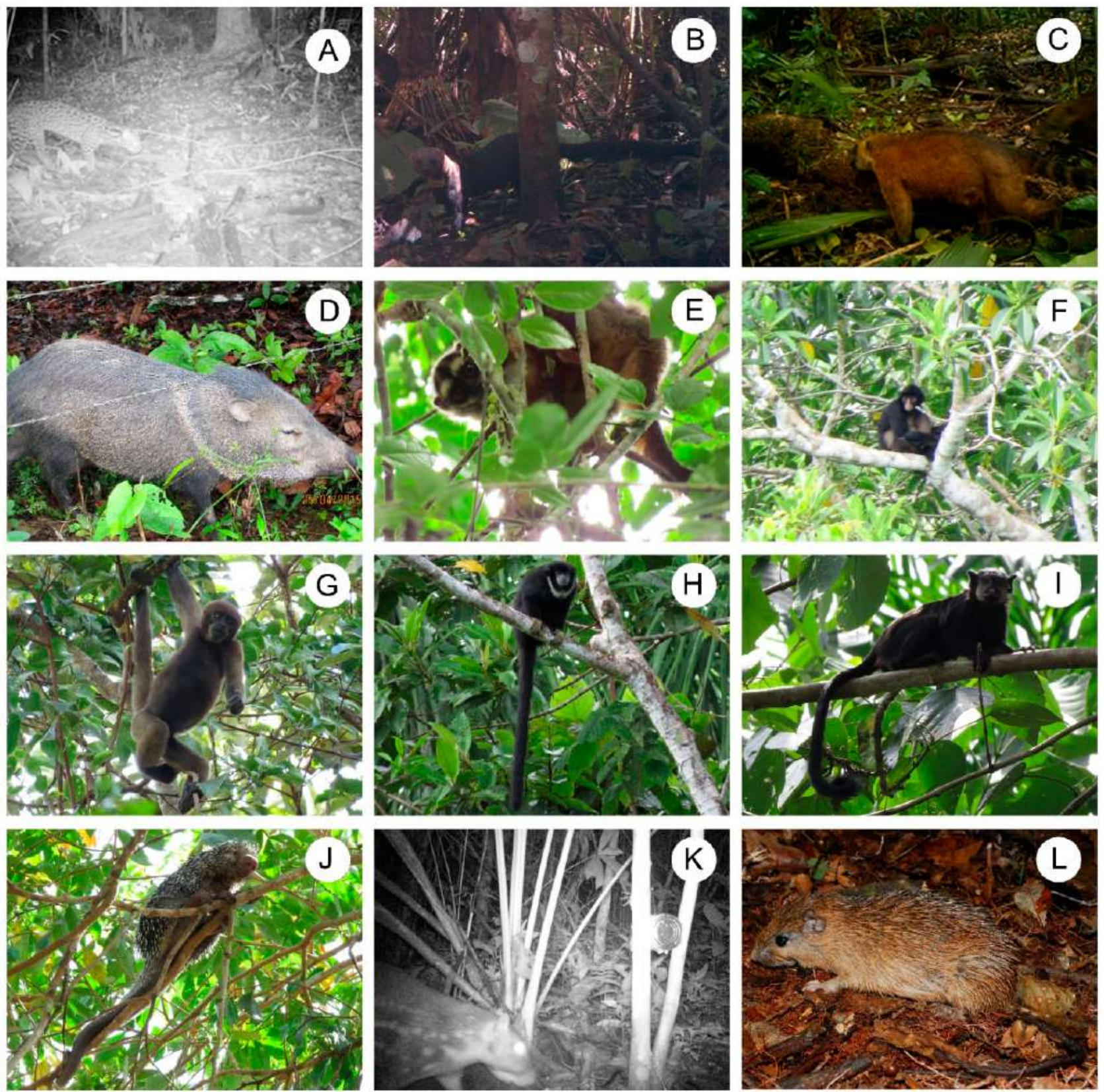

Figura 3. Algunas especies de mamíferos registrados en el municipio de San José del Guaviare, Colombia. A, Leopardus pardalis; B, Eira barbara; C, Nasua nasua; D, Pecari tajacu; E, Aotus vociferans; F, Ateles belzebuth; G, Lagothrix lagothricha; H, Cheracebus lugens; I, Saguinus inustus; J, Coendou prehensilis; K, Cuniculus paca; L, Mesomys hispidus. Fotografías: A, C y L, Instituto SINCHI; B, D y K, Grupo en Conservación y Manejo de Vida Silvestre, Universidad Nacional de Colombia; E-J, Wilmer A. Ramírez.

Figure 3. Some mammal species recorded in the municipality of San José del Guaviare. A, Leopardus pardalis; B, Eira barbara; C, Nasua nasua; D, Pecari tajacu; E, Aotus vociferans; F, Ateles belzebuth; G, Lagothrix lagothricha; H, Cheracebus lugens; I, Saguinus inustus; J, Coendou prehensilis; K, Cuniculus paca; L, Mesomys hispidus. Photographs: A, C y L, Instituto SINCHI; B, D y K, Grupo en Conservación y Manejo de Vida Silvestre, Universidad Nacional de Colombia; E-J, Wilmer A. Ramírez. 
objeto de caza como Puma concolor, Herpailurus yagouaroundi, Leopardus wiedii y Panthera onca, y especies poco comunes como Priodontes maximus, Cabassous unicinctus, Cyclopes didactylus y Atelocynus microtis.

Según las categorías propuestas por la IUCN (2020) se reportan 11 especies con algún grado de amenaza. Ateles belzebuth, I. geoffrensis y Pteronura brasiliensis se encuentran en peligro (EN); ocho especies se encuentran vulnerables (VU) y seis especies casi amenazadas (NT), mientras que 100 especies están categorizadas como preocupación menor (LC; Anexo 1).

Representatividad del muestreo. Para murciélagos capturados en redes de niebla obtuvimos una cobertura del muestreo del $64 \%$ con 778 individuos; para pequeños mamíferos no voladores capturados mediante trampas Sherman alcanzamos una cobertura del muestreo del
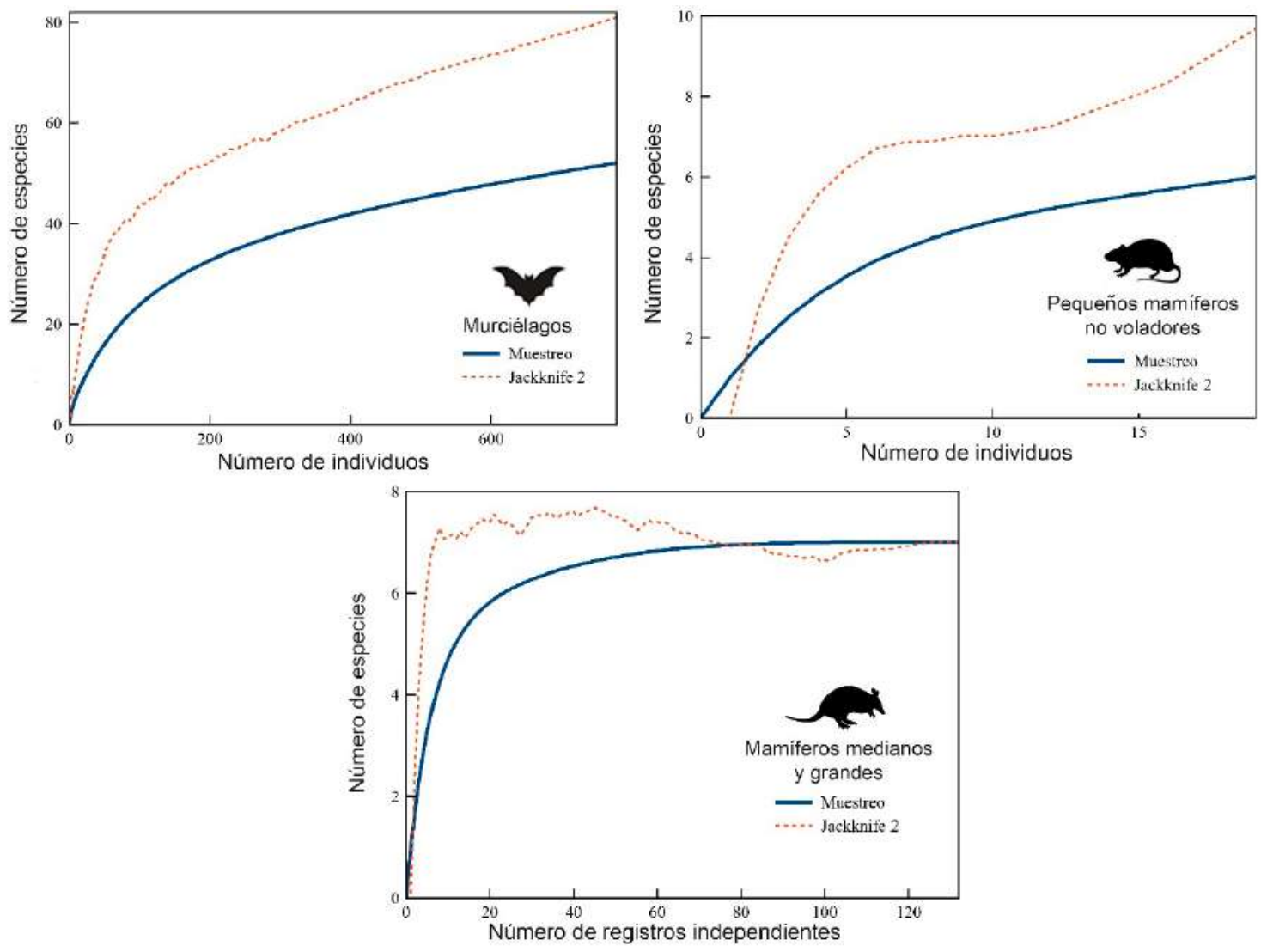

Figura 4. Curvas de acumulación de especies y modelo de Jackknife 2 para murciélagos (redes de niebla), pequeños mamíferos no voladores (trampas Sherman), y mamíferos medianos y grandes (cámaras trampa), en San José del Guaviare, Colombia.

Figure 4. Species accumulation curve and Jackknife 2 model for bats (mist nets), small non volant mammals (Sherman traps), and medium and big mammals (camera traps), in San José del Guaviare, Colombia. 
Novedades geográficas. Presentamos las novedades geográficas resumidas así:

Marmosa waterhousei, corresponde al sexto registro para el país y el segundo para el oriente de los Andes Colombianos (Gutiérrez et al., 2011).

Glironia venusta, reportada recientemente por Montenegro \& Restrepo (2018), corresponde al registro más al norte dentro de su distribución, con una extensión de aproximadamente $420 \mathrm{~km}$ de su única localidad conocida.

Lonchorhina marinkellei es un murciélago que previo a los presentes registros solo se conocía para la localidad tipo en Durania, Cueva Superior, en el departamento del Vaupés, recolectado hace más de 40 años (Hernández-Camacho \& Cadena, 1978). Este registro extendió $320 \mathrm{~km}$ al occidente su distribución y muestra que al parecer la especie está fuertemente asociada a afloramientos del escudo Guayanés (Morales-Martínez \& López-Arévalo, 2018).

Glossophaga commissarisi representa el registro más al norte reportado para la especie; previamente en Colombia solo había sido reportado para Leticia, Amazonas; nuestro registro extiende $750 \mathrm{~km}$ hacia el noroccidente la distribución de la especie (Webster \& Jones, 1993).

Phyllostomus latifolius es una especie con muy pocos registros en Colombia, reportada por última vez por Montenegro \& Romero-Ruiz (1999). Solo se conocían dos localidades para la especie: Mitú en el departamento de Vaupés (Marinkelle \& Cadena, 1972) y el Río Mesay en el departamento de Caquetá (Montenegro \& Romero-Ruiz, 1999). Los registros reportados aquí extienden la distribución cerca de $320 \mathrm{~km}$ al occidente de Mitú y cerca de $280 \mathrm{~km}$ al norte del Río Mesay.

Glyphonycteris daviesi es una especie rara y solo se conoce de tres localidades, en el piedemonte oriental de la Cordillera Oriental, en la altillanura colombiana (Morales-Martínez \& Suárez-Castro, 2014) y en San José del Guaviare (Agudelo-Liz et al., 2018). En nuestro conocimiento solo se sabe de un espécimen para Colombia, proveniente de Tame en el departamento de Arauca (Morales-Martínez \& Suárez-Castro, 2014). Este registro representa la confirmación de la presencia de la especie en San José del Guaviare y el segundo espécimen reportado en Colombia, ya que de las demás localidades solo se ha reportado a partir de fotografías.
Glyphonycteris sylvestris se conocía según la última revisión de su distribución en Colombia en cinco localidades; sin embargo, los especímenes de tres localidades se reportan como extraviados (Morales-Martínez \& Suárez-Castro, 2014). No obstante, los registros extraviados mencionados por estos autores corresponden en realidad a Trinycteris nicefori, por lo cual no fueron encontrados. Por lo tanto, a la fecha la única mención de la especie para el oriente de la cordillera de los Andes es la incluida en Mantilla-Meluk et al. (2017) para el sector norte de la Serranía de Chiribiquete, aunque no se menciona la localidad exacta. En consecuencia, el ejemplar capturado corresponde a la segunda localidad de la especie para la Amazonía colombiana y confirma la presencia de la especie al oriente de los Andes.

Myotis keaysi solamente había sido reportada en Colombia para las tierras altas (por encima de los 1000 m s.n.m.) de la cordillera de los Andes, aunque su distribución incluye las tierras bajas de México, América Central y la isla de Trinidad (Wilson, 2008), y hasta los 500 m.s.n.m. en la vertiente Amazónica de los Andes en Perú (Patterson et al 1996). No existen registros para las tierras bajas del oriente de los Andes en Colombia, siendo este el primer registro para la zona cis-Andina en Colombia, lo que sugiere que posiblemente la especie presente una distribución más amplia en el país.

\section{Discusión}

Con 121 especies nativas, la riqueza de mamíferos encontrada en San José del Guaviare corresponde a un 23.5 \% de los mamíferos reportados en el país (Ramírez-Chaves et al., 2019). Esta cifra es mayor que el número de especies reportadas para localidades muestreadas sistemáticamente en Centroamérica, donde se han reportado 117 especies a lo largo de 34 años para la estación "La Selva", Costa Rica, y 113 especies a lo largo de 71 años en Barro Colorado, Panamá (Voss \& Emmons, 1996). Esta riqueza es también comparable con otros inventarios a mediano y largo plazo en Suramérica, donde se han reportado 120 especies para Manaus, Brasil en un periodo de seis años (Voss \& Emmons, 1996; Sampaio et al., 2003), 102 especies en cuatro años para Paracou, Guyana Francesa (Simmons \& Voss 1998; Voss et al., 2001) y 130 especies en un periodo de siete años para el bosque de Iwokrama, Guyana (Lim \& Engstrom, 2005). Así mismo reportamos un número mayor de especies para San José del Guaviare, en relación a trabajos previos en la zona de estudio, donde se 
habían reportado entre 42 y 51 especies de mamíferos (Agudelo-Liz et al., 2018; Montenegro \& Restrepo 2018; Trujillo et al., 2018).

Para el caso de los murciélagos se obtuvo una mediana representatividad del inventario (64 \%; Figura 4), considerando solamente las capturas en redes de niebla (52 especies). Este valor sumado a las especies registradas por captura manual e información secundaria llegan a 63 especies en el municipio (Anexo 1), cifra menor a la riqueza estimada según Jackknife 2 (81 especies), lo que indica que el inventario aún no está completo. Dado el énfasis en el uso de redes de niebla, existe una probable subestimación en cuanto al número de especies insectívoras de familias como Molossidae, Thyropteridae, Furipteridae y algunas especies de la familia Vespertilionidae que no son frecuentemente capturadas a través de esta metodología (Pech-Canche et al., 2010). Por tanto, consideramos que la diversidad de este grupo debe ser mayor a la encontrada y se requiere del estudio con técnicas complementarias como la detección por ultrasonido para tener una mayor cobertura taxonómica del grupo.

A pesar de que la diversidad de murciélagos en San José del Guaviare pueda estar subestimada, este sitio contiene la riqueza más alta reportada para el país, con 63 especies. Factores como la heterogeneidad en cuanto a tipos de cobertura con bosques amazónicos y afloramientos rocosos en una relativa cercanía (Montenegro \& Romero-Ruiz, 1999), y la disponibilidad de cuevas como refugio, pueden estar relacionados con la riqueza de murciélagos encontrada. En Colombia otros inventarios con gran número de especies de murciélagos corresponden a los realizados en varias localidades del sector norte de La Serranía de la Macarena (Sánchez-Palomino et al., 1993), donde se registraron 44 especies; el inventario en varias localidades del sector sur de la Serranía de Chiribiquete, con 46 especies (Montenegro \& Romero-Ruiz, 1999); varias localidades de ecosistemas de sabanas inundables de Casanare, Orinoquía Colombiana, donde se registraron 50 especies (Morales-Martínez et al., 2018); y el inventario realizado en varias localidades del sector norte de la Serranía de Chiribiquete, en el cual se reportan 56 especies (Mantilla-Meluk et al., 2017).

La riqueza de especies de pequeños mamíferos no voladores es baja, con 13 especies nativas, en comparación con otras zonas por debajo de 1000 m.s.n.m., incluyendo la Amazonía y la Orinoquía, donde se han reportado de 21 a 24 especies (Ramírez-Chaves et al., 2013;
Mora-Fernández \& Rodríguez-Posada, 2017), así como para otras localidades amazónicas como Cuzco, Perú, y Paracou, Guayana Francesa, donde se registran 22 especies (Voss et al., 2001). Los estimadores de capturas por trampas Sherman muestran que nuestro esfuerzo de muestreo no es representativo y estima que documentamos el $60 \%$ de la diversidad. Por lo tanto, se sugiere aumentar el esfuerzo de muestreo para el área, así como el uso de otras técnicas como trampas de caída, las cuales pueden ser más eficientes que las trampas Sherman (Umetsu et al., 2006), y el uso de trampas instaladas en el dosel para la captura de especies arborícolas (Lambert et al., 2005).

Las 11 especies de primates registradas representan el $28.9 \%$ de las especies colombianas y se confirma la presencia de todas las especies probables por distribución para la zona (Defler, 2003). Esta riqueza es mayor comparada con seis comunidades de primates en la Amazonía y tres comunidades de la Orinoquía evaluadas por Defler (2013), en las que registra entre dos y diez especies simpátricas. La mayoría de las especies son de distribución amazónica a excepción de Alouatta seniculus, que tiene una distribución amplia en el país y Plecturocebus ornatus, la cual se encuentra mayormente hacia la Orinoquía (Defler, 2003). Se destaca la presencia de Ateles belzebuth, ya que tiene baja densidad poblacional a lo largo de su distribución y, aunque se ha insinuado su presencia en los rebalses del río Guaviare, son muy pocos los registros que a la fecha que confirman su presencia. Adicionalmente, se confirmó la presencia de Plecturocebus ornatus al sur del río Guaviare, como sugiere Defler (2003).

Nuestros resultados indican que los inventarios de mamíferos realizados en el municipio de San José del Guaviare no están completos y se esperan más especies de las registradas en los dos órdenes más diversos de mamíferos en Colombia (Chiroptera y Rodentia), lo que permite inferir que la riqueza presentada aquí está subestimada. No obstante, el conocimiento de la diversidad biológica es necesario para la evaluación de las prioridades de investigación y conservación de los bosques húmedos en Suramérica (Voss \& Emmons, 1996). Por consiguiente, es primordial seguir implementando inventarios en el municipio y complementar este tipo de estudios a largo plazo en otras localidades de Colombia. Para ello es recomendable continuar con la recolección de especímenes biológicos que se depositen en colecciones biológicas de instituciones reconocidas, ya que permiten conocer la variabilidad de las especies, describir la diversidad aún desconocida, conocer la distribución 
de especies en peligro y sus amenazas, hacer comparaciones entre inventarios, y finalmente permiten establecer con certeza la riqueza de un sitio, la cual es el parámetro básico en los estudios de la biodiversidad y en muchos casos la única información disponible para la toma de decisiones ambientales (Pineda-López , 2019).

Amenazas y oportunidades para la conservación de mamíferos del municipio. La situación ambiental actual del departamento del Guaviare es preocupante, ya que a pesar de tener una gran biodiversidad, está inmerso en uno de los focos principales de deforestación del país. Entre 2018 y 2019, el municipio de San José del Guaviare ha presentado entre el $4 \%$ y el $10 \%$ de las detecciones tempranas de deforestación en Colombia (IDEAM, 2018, 2019). Esto ha provocado una disminución drástica de la conectividad entre los ecosistemas, principalmente en la zona que hace parte del área de Manejo Especial de la Macarena (AMEM; ANLA 2017) inducido por el establecimiento de pastizales para usurpación de tierras o actividades ganaderas, cultivos ilícitos y la expansión de infraestructura vial informal. A esto se le suma el reciente plan del gobierno colombiano de la construcción de una vía de $381 \mathrm{~km}$ que conectaría San José del Guaviare con San Vicente del Caguán, Caquetá, lo cual incrementaría la fragmentación del hábitat y la pérdida de conectividad (Clerici et al., 2018); por tanto, representa una seria amenaza para los mamíferos y requiere de acciones de conservación urgentes. Por ello, estrategias de turismo rural sostenible en las Reservas de la Sociedad Civil, áreas de reserva forestal y fincas agroecológicas, son una alternativa económica sustentable para los pobladores que garantizan la conservación de la biodiversidad.

\section{Agradecimientos}

Agradecemos principalmente a la Universidad Nacional de Colombia por seguir incentivando y proporcionando las herramientas necesarias para continuar con la investigación sobre la fauna colombiana. Especialmente agradecemos a Wilmer Andrés Ramírez Riaño, poblador local de San José del Guaviare por sus observaciones y fotografías sobre mamíferos de las veredas, a los pobladores de Playa Güio por toda su ayuda y colaboración; así como a los habitantes de las diferentes localidades visitadas. La información recolectada por parte del Instituto SINCHI fue parte del proyecto "Conservación y aprovechamiento sostenible de la diversidad biológica, socioeconómica y cultural de la Amazonía colombiana" desarrollado por el Instituto Amazónico de Investigaciones Científicas SINCHI. Finalmente agradecemos a los evaluadores por sus comentarios y aportes que fueron de ayuda para mejorar el manuscrito. Los especímenes recolectados del 2012 al 2014, fueron recolectados bajo el Permiso Marco de Colecta Científica de la Universidad Nacional de Colombia y la resolución No. DSGV-112 de octubre 25 de 2012 emitida por la Corporación para el Desarrollo Sostenible del Norte y Oriente Amazónico, mientras que las colectas del 2017 realizadas por el Instituto Amazónico de Investigaciones Científicas SINCHI no requieren de un permiso de colecta al tratarse de una entidad científica adscrita y vinculada al Sistema Nacional Ambiental (SINA).

\section{Referencias}

Agudelo-Liz, R., Giraldo-Gutiérrez, V. \& Setina-Liz, V. H. (2018). Murciélagos de San José del Guaviare. Field Guides of Field Mususeum, 1006, 1-5.

ANLA. (2017). Reporte área de manejo especial de la Macarena. Bogotá D. C.: Subdirección de Instrumentos, Permisos y Trámites Ambientales, Autoridad Nacional de Licencias Ambientales.

Barona-Colmenares, A. A., Contreras-Herrera, J., Vriesendorp, C. \& Cárdenas-López, D. (2018). Vegetación y flora. En: Vriesendorp, C., Pitman, N., Alvira Reyes, D., Salazar Molano, A., Botero-García, R., Arciniegas, A., de Souza, L., del Campo, Á., Stotz, D. F., Wachter, T., Ravikumar, A. \& Peplinski, J., (Eds.). La Lindosa, Capricho, Cerritos. Rapid Biological and Social Inventories Report 29. (pp. 198-210). Chicago, USA: The Field Museum, Colombia.

Clerici, N., Salazar, C., Pardo-Díaz, C., Jiggins, C. D., Richardson, J. E. \& Linares, M. (2018). Peace in Colombia is a critical moment for Neotropical connectivity and conservation: Save the northern Andes-Amazon biodiversity bridge. Conservation Letters, 12, 1-7. https:/ / doi.org/10.1111/conl.12594

Colwell, R. K. 2013. EstimateS: Statistical estimation of species richness and shared species from samples. Version 9. Disponible en:

http:/ / purl.oclc.org/estimates.

Defler, T. R. (2003). Primates de Colombia. Bogotá D.C.: Conservación Internacional Colombia.

Defler, T. R. (2013). Species richness, densities and biomass of nine primate communities in eastern Colombia. Revista de la Academia Colombiana de Ciencias Exactas Físicas y Naturales, 37(143), 253-262.

https:// doi.org/10.18257/raccefyn.8 
Emmons, L. \& Feer, F. (1997). Neotropical rainforest mammals: a field guide. Chicago, USA: University of Chicago Press.

Gotelli, N. J., \& Colwell, R. K. (2011). Estimating species richness. Frontiers in Measuring Biodiversity. New York (NY): Oxford University Press.

Gutiérrez, E. E., Soriano, P. J., Rossi, R. V., Murillo, J. J., Ochoa-G. J. \& Aguilera, M. (2011). Occurrence of Marmosa waterhousei in the Venezuelan Andes, with comments on its biogeographic significance. Mammalia, 75, 381-386.

Heltshe, J. F., \& Forrester, N. E. (1983). Estimating diversity using quadrat sampling. Biometrics, 39(4), 1073-1076.

Hernández-Camacho, J. \& Cadena, A. (1978). Notas para la revisión del género Lonchorhina (Chiroptera, Phyllostomidae). Caldasia, 12(57), 199-251.

Hernández Camacho, J. I., Hurtado-Guerra, A., Ortiz-Quijano, R. \& Walschburger, T. (1992). Unidades biogeográficas de Colombia. En Halffter, G. (Ed.). La diversidad biológica de Iberoamérica I. (pp. 105-152). Xalapa: Acta Zoológica Mexicana, Instituto de Ecología, A.C.

IDEAM. (2018). Boletín de alertas tempranas de deforestación (AT-D) 17 cuarto trimestre octubre-diciembre 2018. Bogotá D. C.: Instituto de Hidrología, Meteorología y Estudios Ambientales.

IDEAM. (2019). Boletín de alertas tempranas de deforestación (AT-D) 21 cuarto trimestre octubre-diciembre 2019. Bogotá D. C.: Instituto de Hidrología, Meteorología y Estudios Ambientales.

IUCN. International Union for Conservation of Nature (2020). The IUCN Red List of Threatened Species. Version 2020-1. Disponible en

http:/ / www.iucnredlist.org.

Jiménez-Ramírez, J. S. (2014). Inventario de especies de murciélagos y análisis preliminar de dietas de los pertenecientes al gremio frugivoro en reservas privadas de la vereda Playa Güio, San José del Guaviare. (Trabajo de grado). Bogotá D. C.: Universidad Nacional de Colombia, Facultad de Ciencias, Departamento de Biología.

Lambert, T. D., Malcolm, J. R. \& Zimmerman, B. L. (2005). Variation in small mammal species richness by trap height and trap type in southeastern Amazonia. Journal of Mammalogy, 86(5), 982-990.

https:/ / doi.org/10.1644/1545-1542(2005)86[982:VISMSR]2.0.CO;2

Lim, B. K. \& Engstrom, M. D. (2005). Mammals of Iwokrama forest. Proceedings of the Academy of $\mathrm{Na}$ tural Sciences of Philadelphia, 154(1), 71-108.

https:/ / doi.org/10.1635/0097-3157(2004)154[0071:$\mathrm{MOIF}] 2.0 . \mathrm{CO} ; 2$
López-Arévalo, H. F., Carillo-Villamizar, J. Z., Díaz-Rodríguez, J. V. \& Delgadillo-Ordóñez, N. C. (2019). Guía de mamíferos de San José del Guaviare. Bogotá D.C.: Universidad Nacional de Colombia.

Mantilla-Meluk, H., Mosquera-Guerra, F., Trujillo, F., Pérez, N., Velásquez-Valencia, A. \& Vargas-Perez, A. (2017). Mamíferos del sector norte del parque Nacional Natural Serranía de Chiribiquete. Revista Colombia Amazónica, 10, 99-134.

Marinkelle, C. J. \& Cadena, A. (1972). Notes on bats new to the fauna of Colombia. Mammalia, 36, 50-58.

https:/ / doi.org/10.1515/mamm.1972.36.1.50

Ministerio de Ambiente y Desarrollo Sostenible. (15 de septiembre de 2017). Por la cual se establece el listado de las especies silvestres amenazadas de la diversidad biológica colombiana continental y marino-costera que se encuentran en el territorio nacional, y se dictan otras disposiciones. [Resolución 1912 de 15 de septiembre de 2017].

Montenegro, O. L. \& Romero-Ruíz, M. (1999). Murciélagos del sector sur de la serranía de Chiribiquete, Caquetá, Colombia. Revista de la Academia Colombiana de Ciencias, 23(Supl.), 641-649.

Montenegro, O. L. \& Restrepo, H. (2018). Mamíferos. En Vriesendorp, C., Pitman, N., Alvira-Reyes, D., Salazar-Molano, A., Botero-García, R., Arciniegas, A., de Souza, L., del Campo, Á., Stotz, D. F., Wachter, T., Ravikumar, A. \& Peplinski, J., (Eds.). La Lindosa, Capricho, Cerritos. Rapid Biological and Social Inventories Report 29 (pp. 139-146. Chicago, USA: The Field Museum.

Mora-Fernández, C. \& Rodríguez-Posada, M. E. (2017). Fauna y flora del Casanare de la montaña a la sabana: una visión integral del territorio. Yopal, Colombia: Gobernación de Casanare.

Morales-Martínez, D. M. \& Suárez-Castro, A. F. (2014). New records for Glyphonycteris Thomas, 1896 (Chiroptera: Phyllostomidae) from Colombia. Check List, $10,639-644$.

https:/ / doi.org/10.15560/10.3.639

Morales-Martínez, D. M. \& López-Arévalo, H. F. (2018). Distribución y conservación de los murciélagos del género Lonchorhina (Chiroptera: Phyllostomidae) en Colombia. Caldasia, 40(2), 349-365.

https:/ / doi.org/10.15446/caldasia.v40n2.70415

Morales-Martínez, D. M., Rodríguez-Posada, M. E., Fernández-Rodríguez, C., Calderón-Capote, M. C. \& Gutiérrez-Sanabria, D. R. (2018). Spatial variation of bat diversity between three floodplain-savanna ecosystems of the Colombian Llanos. Therya, 9(1), 41-52.

https:/ / doi.org/10.12933/ therya-18-537 
Patterson, B. D., Pacheco, V., \& Solari, S. (1996). Distributions of bats along an elevational gradient in the Andes of southeastern Peru. Journal of Zoology, 240, 237-658.

https:// doi.org/10.1111/j.1469-7998.1996.tb05313.x

Pech-Canche, J. M., MacSwiney, G. C, \& Estrella, E. 2010. Importancia de los detectores ultrasónicos para mejorar los inventarios de murciélagos Neotropicales. Therya. 1(3), 221-228.

https:/ / doi.org/10.12933/therya-10-17

Pineda-López, R. (2019). Estimadores de la riqueza de especies. En: Moreno C. E. (Ed.). La biodiversidad en un mundo cambiante: Fundamentos teóricos y metodológicos para su estudio. Pp: 159-174. Ciudad de México: Universidad Autónoma del Estado de Hidalgo, Libermex.

Ramírez-Chaves, H. E., Noguera-Urbano, E. A. \& Rodríguez-Posada, M. E. (2013). Mamíferos (Mammalia) del departamento de Putumayo, Colombia. Revista de la Academia Colombiana de Ciencias, 37(143), 263-286. https:/ / doi.org/10.18257/ raccefyn.9

Ramírez-Chaves, H. E, Suárez Castro, A. F., Sociedad Colombiana de Mastozoología, Zurc, D. Concha-Osbahr, D. C., Trujillo, A., Noguera Urbano, E. A., Pantoja Peña, G. E., Rodríguez Posada, M. E., González Maya, J. F., Pérez Torres, J., Mantilla Meluk, H., López Castañeda, C., Velásquez Valencia, A., \& Zárrate Charry, D. (2019). Mamíferos de Colombia. Version 1.6. Sociedad Colombiana de Mastozoología. Checklist dataset

https:/ / doi.org/10.15472/kl1whs accessed via GBIF. org on 2019-08-18.

Redondo, R. A. F., Brina, L. P. S, Silva, R. F., Ditchfield, A. D. \& Santos, F. R. (2008). Molecular systematics of the genus Artibeus (Chiroptera: Phyllostomidae). Molecular Phylogenetics and Evolution, 49(1), 44-58.

https:/ / doi.org/10.1016/j.ympev.2008.07.001

Rodríguez-Castellanos, P., Botero-Cruz, A. M. \& Cruz-Antia, D. (2013). Los felinos y la gente de la Serranía de la Lindosa. Compartiendo territorio. Bogotá D. C.: CDA, Fundación Omacha-Fundación Panthera.

Sampaio, E. M., Kalko, E. K., Bernard, E., Rodríguez-Herrera, B. \& Handley, C. O. (2003). A biodiversity assessment of bats (Chiroptera) in a tropical lowland rainforest of Central Amazonia, including methodological and conservation considerations. Studies on Neotropical Fauna and Environment, 38(1), 17-31.

https:/ / doi.org/10.1076/snfe.38.1.17.14035

Sánchez, F. Sánchez-Palomino, P. \& Cadena, A. (2004). Inventario de mamíferos en un bosque de Los Andes centrales de Colombia. Caldasia, 26(1), 291-309.
Sánchez-Palomino, P., Rivas-Pava, P. \& Cadena, A. (1993). Composición, abundancia y riqueza de especies de la comunidad de murciélagos en bosques de galería en la Serranía de La Macarena (Meta-Colombia). Caldasia, 17(2), 301-312.

Simmons, N. B. \& Voss, R. S. (1998). The mammals of Paracou, Frence Guiana: A Neotropical lowland rainforest fauna part 1. Bats. Bulletin of the American Museum of Natural History, 219, 1-219.

Solari, S., Pacheco, V., Luna, L., Velazco, P. M. \& Patterson, B. D. (2006). Mammals of the Manu Biosphere Reserve. Fieldiana Zoology, 110, 13-22.

https:// doi.org/10.3158/0015-0754(2006)110[13:MOTMBR]2.0.CO;2

Trujillo, F., Mosquera-Guerra, F., Diaz-Pulido, A., Carvajal-Castro, J. D. \& Mantilla-Meluk. H. (2018). Mamíferos de la Guayana colombiana. En Lasso, C. A., \& Señaris, J. C. (Eds.). Fauna Silvestre del Escudo Guayanés (Colombia-Venezuela) (pp. 345-379). Bogotá D. C.: Instituto de Investigación de Recursos Biológicos Alexander von Humboldt.

Umetsu, F., Naxara, L. \& Pardini, R. (2006). Evaluating the efficiency of pitfall traps for sampling small mammals in the Neotropics. Journal of Mammalogy, 87(4), 757-765.

https:/ / doi.org/10.1644/05-MAMM-A-285R2.1

Voss, R. S. \& Emmons, L. (1996). Mammalian diversity in Neotropical lowland rainforests: a preliminary assessment. Bulletin of the American Museum of Natural History, 230, 1-115.

Voss, R. S., Lunde, D. P. \& Simmons, N. B. (2001). The Mammals of Paracou, French Guiana: a Neotropical Lowland Rainforest Fauna Part 2. Nonvolant Species. Bulletin of the American Museum of Natural History, 236, 1-263.

https:/ / doi.org/10.1206/0003-0090(2001)263<0003:T$\mathrm{MOPFG}>2.0 . \mathrm{CO} ; 2$

Webster, W. \& Jones, J. K. (1993). Glossophaga commissarisi. Mammalian Species, (446), 1-4.

https:/ / doi.org/10.2307/3504303

Willott, S. J. (2001). Species accumulation curves and the measure of sampling effort. Journal of Applied Ecology, 38(2), 484-486.

https:/ / doi.org/10.1046/j.1365-2664.2001.00589.x

Wilson, D. E. (2008). Genus Myotis Kaup, 1829. En Gardner, A. L. (Ed.). Mammals of South America, Volume I: Marsupials, xenarthrans, shrews, and bats (pp. 468-481). Chicago: The University of Chicago Press. 


\section{Anexos}

Anexo 1. Listado de especies de mamíferos presentes en el municipio de San José del Guaviare, Colombia con la categoría de amenaza para cada especie y el método de captura de cada una. CO, recolección ocasional; CT, cámara trampa; E, entrevista; M, recolección manual; O, observación; R, red de niebla; TS, trampa Sherman; TT, trampa Tomahawk.

\begin{tabular}{|c|c|c|c|c|}
\hline Especie & IUCN & $\begin{array}{l}\text { Resolución } \\
\text { 1912-2017 }\end{array}$ & $\begin{array}{l}\text { Método de } \\
\text { captura }\end{array}$ & Otros $^{A}$ \\
\hline \multicolumn{5}{|c|}{ DIDELPHIMORPHIA } \\
\hline Caluromys lanatus (Olfers, 1818) & $\mathrm{LC}$ & - & $\mathrm{O}$ & 1 \\
\hline Glironia venusta Thomas, 1912 & $\mathrm{LC}$ & - & $\mathrm{O}$ & 1 \\
\hline Didelphis marsupialis Linnaeus, 1758 & LC & - & $\mathrm{TT}, \mathrm{CT}, \mathrm{E}$ & 1 \\
\hline Philander andersoni (Osgood, 1913) & $\mathrm{LC}$ & - & $\mathrm{TS}, \mathrm{CT}$ & \\
\hline Marmosa demerarae (Thomas, 1905) & LC & - & TS & \\
\hline Marmosa waterhousei (Tomes, 1860) & - & - & M & \\
\hline \multicolumn{5}{|c|}{ CINGULATA } \\
\hline Dasypus novemcinctus Linnaeus, 1758 & $\mathrm{LC}$ & - & $\mathrm{O}, \mathrm{CT}, \mathrm{E}$ & 1 \\
\hline Dasypus sabanicola Mondolfi, 1968 & NT & - & $\mathrm{O}, \mathrm{E}$ & \\
\hline Cabassous unicinctus (Miller, 1899) & $\mathrm{LC}$ & - & $\mathrm{E}$ & \\
\hline Priodontes maximus (Kerr, 1792) & VU & $\mathrm{EN}$ & $\mathrm{E}$ & \\
\hline \multicolumn{5}{|c|}{ PILOSA } \\
\hline Cyclopes didactylus (Linnaeus, 1758) & $\mathrm{LC}$ & - & $\mathrm{E}$ & 1 \\
\hline Tamandua tetradactyla (Linnaeus, 1758) & $\mathrm{LC}$ & - & $\mathrm{CT}, \mathrm{E}$ & 1,2 \\
\hline Myrmecophaga tridactyla Linnaeus, 1758 & VU & VU & $\mathrm{CT}, \mathrm{E}$ & 1 \\
\hline Bradypus variegatus Schinz, 1825 & $\mathrm{LC}$ & - & $\mathrm{O}, \mathrm{E}$ & 1 \\
\hline Choloepus didactylus (Linnaeus, 1758) & $\mathrm{LC}$ & - & $\mathrm{O}, \mathrm{E}, \mathrm{CO}$ & 1 \\
\hline \multicolumn{5}{|c|}{ PRIMATES } \\
\hline Saguinus inustus (Schwartz, 1951) & LC & - & $\mathrm{O}$ & 1 \\
\hline Cebus albifrons (Humboldt, 1812) & $\mathrm{LC}$ & - & $\mathrm{O}, \mathrm{CO}$ & 1 \\
\hline Sapajus apella (Linnaeus, 1758) & $\mathrm{LC}$ & - & $\mathrm{O}$ & \\
\hline Saimiri cassiquiarensis Lesson, 1840 & $\mathrm{LC}$ & - & $\mathrm{O}, \mathrm{E}$ & 1 \\
\hline Aotus vociferans (Spix, 1823) & $\mathrm{LC}$ & - & $\mathrm{O}$ & \\
\hline Cheracebus lugens (Humboldt, 1811) & $\mathrm{LC}$ & - & $\mathrm{O}$ & \\
\hline Plecturocebus ornatus (Gray, 1866) & VU & VU & $\mathrm{O}$ & \\
\hline Cacajao melanocephalus Humboldt, 1812 & $\mathrm{LC}$ & - & $\mathrm{O}$ & \\
\hline Alouatta seniculus Linnaeus, 1766 & $\mathrm{LC}$ & - & $\mathrm{O}, \mathrm{CO}$ & 1 \\
\hline
\end{tabular}




\begin{tabular}{|c|c|c|c|c|}
\hline Especie & IUCN & $\begin{array}{l}\text { Resolución } \\
\text { 1912-2017 }\end{array}$ & $\begin{array}{l}\text { Método de } \\
\text { captura }\end{array}$ & Otros $^{A}$ \\
\hline Ateles belzebuth É. Geoffroy Saint Hilaire, 1806 & $\mathrm{EN}$ & VU & $\mathrm{O}, \mathrm{E}$ & 1 \\
\hline \multirow[t]{2}{*}{ Lagothrix lagothricha Humboldt, 1812} & VU & VU & $\mathrm{O}, \mathrm{CO}, \mathrm{E}$ & 1 \\
\hline & \multicolumn{2}{|c|}{ CHIROPTERA } & & \\
\hline Cormura brevirostris (Wagner, 1843) & LC & - & $\mathrm{R}$ & 3 \\
\hline Peropteryx macrotis (Wagner, 1843) & LC & - & $\mathrm{R}$ & 3 \\
\hline Rhynchonycteris naso (Wied-Neuwied, 1820) & LC & - & $\mathrm{R}$ & \\
\hline Saccopteryx bilineata (Temminck, 1838) & LC & - & $\mathrm{R}$ & 3 \\
\hline Saccopteryx canescens Thomas, 1901 & LC & - & $\mathrm{R}$ & \\
\hline Saccopteryx leptura (Schreber, 1774) & LC & - & $\mathrm{R}$ & 3 \\
\hline Micronycteris hirsuta (Peters, 1869) & LC & - & $\mathrm{R}$ & 3 \\
\hline Micronycteris megalotis (Gray, 1842) & LC & - & $\mathrm{R}$ & \\
\hline Lampronycteris brachyotis (Dobson, 1879) & LC & - & $\mathrm{R}$ & \\
\hline Desmodus rotundus (É. Geoffroy Saint Hilaire, 1810) & LC & - & $\mathrm{R}$ & 3 \\
\hline Diaemus youngi (Jentink, 1893) & LC & - & & 3 \\
\hline Lonchorhina aurita Tomes, 1863 & LC & - & $\mathrm{R}$ & \\
\hline $\begin{array}{l}\text { Lonchorhina marinkellei Hernández-Camacho \& Ca- } \\
\text { dena, } 1978\end{array}$ & VU & - & $\mathrm{R}$ & \\
\hline Lonchorhina orinocensis Linares \& Ojasti, 1971 & VU & - & $\mathrm{R}$ & \\
\hline $\begin{array}{l}\text { Gardnerycteris crenulatum (É. Geoffroy Saint Hilai- } \\
\text { re, 1803) }\end{array}$ & LC & - & $\mathrm{R}$ & 3 \\
\hline Lophostoma brasiliense Peters, 1867 & LC & - & $\mathrm{R}$ & \\
\hline Lophostoma silvicolum d'Orbigny, 1836 & LC & - & $\mathrm{R}$ & 3 \\
\hline Phylloderma stenops Peters, 1865 & LC & - & $\mathrm{R}$ & 3 \\
\hline Phyllostomus discolor (Wagner, 1843) & LC & - & $\mathrm{R}$ & 3 \\
\hline $\begin{array}{l}\text { Phyllostomus elongatus (É. Geoffroy Saint Hilaire, } \\
\text { 1810) }\end{array}$ & LC & - & $\mathrm{R}$ & \\
\hline Phyllostomus hastatus (Pallas, 1767) & LC & - & $\mathrm{R}$ & 3 \\
\hline Phyllostomus latifolius (Thomas, 1901) & LC & - & $\mathrm{R}$ & \\
\hline Tonatia maresi Williams, Willig \& Reid, 1995 & LC & - & $\mathrm{R}$ & 3 \\
\hline Trachops cirrhosus (Spix, 1823) & LC & - & $\mathrm{R}$ & 3 \\
\hline Glossophaga commissarisi Gardner (1962) & LC & - & $\mathrm{R}$ & \\
\hline Glossophaga soricina (Pallas, 1766) & LC & - & $\mathrm{R}$ & \\
\hline Anoura geoffroyi Gray, 1838 & LC & - & $\mathrm{R}$ & 3 \\
\hline
\end{tabular}




\begin{tabular}{|c|c|c|c|c|}
\hline Especie & IUCN & $\begin{array}{c}\text { Resolución } \\
\text { 1912-2017 }\end{array}$ & $\begin{array}{l}\text { Método de } \\
\text { captura }\end{array}$ & Otros $^{A}$ \\
\hline Lichonycteris degener Miller, 1931 & $\mathrm{LC}$ & - & & $\mathrm{ICN}$ \\
\hline Lionycteris spurrelli Thomas, 1913 & LC & - & $\mathrm{R}$ & \\
\hline Hsunycteris thomasi (J.A Allen, 1904) & $\mathrm{LC}$ & - & $\mathrm{R}$ & \\
\hline Carollia brevicauda (Schinz, 1821) & LC & - & $\mathrm{R}$ & 3 \\
\hline Carollia castanea H. Allen, 1890 & LC & - & $\mathrm{R}$ & 3 \\
\hline Carollia perspicillata (Linnaeus, 1758) & LC & - & $\mathrm{R}$ & 3 \\
\hline Glyphonycteris daviesi (Hill 1964) & LC & - & $\mathrm{R}$ & 3 \\
\hline Glyphonycteris sylvestris Thomas, 1896 & LC & - & $\mathrm{R}$ & \\
\hline Trinycteris nicefori (Sanborn, 1949) & $\mathrm{LC}$ & - & $\mathrm{R}$ & \\
\hline Artibeus lituratus (Olfers, 1818) & LC & - & $\mathrm{R}$ & 3 \\
\hline Artibeus obscurus (Schinz, 1821) & LC & - & $\mathrm{R}$ & 3 \\
\hline Artibeus planirostris (Spix, 1823) & LC & - & $\mathrm{R}$ & 3 \\
\hline Dermanura anderseni (Osgood, 1916) & LC & - & $\mathrm{R}$ & \\
\hline Dermanura gnoma (Handley, 1987) & LC & - & $\mathrm{R}$ & \\
\hline Mesophylla macconnelli Thomas, 1901 & LC & - & $\mathrm{R}$ & 3 \\
\hline Platyrrhinus brachycephalus (Rouk \& Carter, 1972) & LC & - & $\mathrm{R}$ & \\
\hline Platyrrhinus helleri (Peters, 1866) & LC & - & $\mathrm{R}$ & \\
\hline Platyrrhinus infuscus (Peters, 1880) & LC & - & $\mathrm{R}$ & \\
\hline Sphaeronycteris toxophyllum Peters, 1882 & $\mathrm{LC}$ & - & & 3 \\
\hline Sturnira giannae Velazco \& Patterson 2019 & LC & - & $\mathrm{R}$ & 3 \\
\hline Sturnira tildae de la Torre, 1959 & LC & - & $\mathrm{R}$ & \\
\hline Uroderma bilobatum Peters, 1866 & LC & - & $\mathrm{R}$ & \\
\hline Uroderma magnirostrum Davis, 1968 & $\mathrm{LC}$ & - & $\mathrm{R}$ & \\
\hline Vampyressa thyone Thomas, 1909 & LC & - & $\mathrm{R}$ & \\
\hline Thyroptera tricolor Spix, 1823 & LC & - & & 3 \\
\hline Eumops auripendulus (G. Shaw, 1800) & LC & - & & ICN \\
\hline Molossus molossus (Pallas, 1766) & LC & - & $\mathrm{R}$ & 3 \\
\hline Molossus rufus É. Geoffroy Saint Hilaire, 1805 & LC & - & $\mathrm{R}$ & \\
\hline Eptesicus brasiliensis (Desmarest, 1819) & LC & - & $\mathrm{R}$ & \\
\hline Eptesicus chiriquinus Thomas, 1920 & $\mathrm{LC}$ & - & $\mathrm{R}$ & \\
\hline Eptesicus furinalis (D’Orbigny \& Gervais, 1847) & $\mathrm{LC}$ & - & & 3 \\
\hline Lasiurus blossevillii (Lesson, 1826) & $\mathrm{LC}$ & - & & 3 \\
\hline
\end{tabular}




\begin{tabular}{|c|c|c|c|c|}
\hline Especie & IUCN & $\begin{array}{c}\text { Resolución } \\
\text { 1912-2017 }\end{array}$ & $\begin{array}{l}\text { Método de } \\
\text { captura }\end{array}$ & Otros $^{A}$ \\
\hline Myotis keaysi J.A. Allen, 1914 & LC & - & $\mathrm{R}$ & \\
\hline Myotis nigricans (Schinz, 1821) & LC & - & & 3 \\
\hline Myotis riparius Handley, 1960 & LC & - & $\mathrm{R}$ & \\
\hline \multirow[t]{2}{*}{ Rhogeessa io Thomas, 1903} & LC & - & $\mathrm{R}$ & \\
\hline & \multicolumn{2}{|c|}{ CARNIVORA } & & \\
\hline Leopardus pardalis (Linnaeus, 1758) & LC & - & $\mathrm{E}, \mathrm{CT}, \mathrm{CO}$ & 2 \\
\hline Leopardus wiedii (Schinz, 1821) & NT & - & $\mathrm{E}$ & 1,2 \\
\hline Puma concolor (Linnaeus, 1771) & LC & - & $\mathrm{E}$ & 1,2 \\
\hline $\begin{array}{l}\text { Herpailurus yagouaroundi (É. Geoffroy Sant-Hilai- } \\
\text { re, 1803) }\end{array}$ & LC & - & E & 1,2 \\
\hline Panthera onca (Linnaeus, 1758) & NT & VU & E & 1,2 \\
\hline Atelocynus microtis (Sclater, 1883) & NT & - & E & \\
\hline Cerdocyon thous (Linnaeus, 1766) & LC & - & $\mathrm{O}, \mathrm{E}$ & \\
\hline Speothos venaticus (Lund, 1842) & NT & - & & 2 \\
\hline Lontra longicaudis (Olfers, 1818) & NT & VU & $\mathrm{O}$ & 1 \\
\hline Pteronura brasiliensis (Gmelin, 1788) & EN & EN & E & \\
\hline Eira barbara (Linnaeus, 1758) & LC & - & $\mathrm{O}, \mathrm{CT}, \mathrm{E}$ & 1 \\
\hline Nasua nasua (Linnaeus, 1766) & LC & - & $\mathrm{O}, \mathrm{CT}$ & \\
\hline \multirow[t]{2}{*}{ Procyon cancrivorus (G. Cuvier, 1798) } & LC & - & CT & \\
\hline & \multicolumn{2}{|c|}{ PERISSODACTYLA } & & \\
\hline \multirow[t]{2}{*}{ Tapirus terrestris (Linnaeus, 1758) } & VU & $\mathrm{CR}$ & $\mathrm{CT}, \mathrm{E}$ & 1 \\
\hline & \multicolumn{2}{|c|}{ ARTIODACTYLA } & & \\
\hline Pecari tajacu (Linnaeus, 1758) & LC & - & $\mathrm{CT} ; \mathrm{O}, \mathrm{E}$ & 1,2 \\
\hline Tayassu pecari (Link, 1795) & VU & - & $\mathrm{CT}, \mathrm{CO}, \mathrm{E}$ & 1,2 \\
\hline Odocoileus virginianus Zimmermann, 1780 & LC & - & $\mathrm{O}, \mathrm{E}$ & 1 \\
\hline \multirow[t]{2}{*}{ Mazama nemorivaga F. Cuvier, 1817} & $\mathrm{LC}$ & - & & 1,2 \\
\hline & \multicolumn{2}{|c|}{ CETACEA } & & \\
\hline \multirow[t]{2}{*}{ Inia geoffrensis (Blainville, 1817) } & EN & VU & $\mathrm{O}, \mathrm{E}$ & \\
\hline & \multicolumn{2}{|c|}{ RODENTIA } & & \\
\hline Hadrosciurus igniventris (Wagner, 1842) & LC & - & $\mathrm{O}, \mathrm{E}, \mathrm{CT}$ & \\
\hline Microsciurus flaviventer (Gray, 1867) & LC & - & $\mathrm{O}$ & \\
\hline
\end{tabular}




\begin{tabular}{|c|c|c|c|c|}
\hline Especie & IUCN & $\begin{array}{c}\text { Resolución } \\
\text { 1912-2017 }\end{array}$ & $\begin{array}{l}\text { Método de } \\
\text { captura }\end{array}$ & Otros $^{A}$ \\
\hline Hylaeamys sp. & - & - & TS & \\
\hline Neacomys spinosus (Thomas, 1882) & $\mathrm{LC}$ & - & TS & \\
\hline Oecomys bicolor (Tomes, 1860) & $\mathrm{LC}$ & - & TS & \\
\hline Hydrochoerus hydrochaeris (Linnaeus, 1766) & $\mathrm{LC}$ & - & $\mathrm{O}, \mathrm{E}$ & \\
\hline Cuniculus paca (Linnaeus, 1766) & $\mathrm{LC}$ & - & $\mathrm{O}, \mathrm{CT}, \mathrm{E}$ & 1,2 \\
\hline Dasyprocta fuliginosa Wagler, 1832 & $\mathrm{LC}$ & - & $\mathrm{CT}, \mathrm{O}, \mathrm{E}$ & 1,2 \\
\hline Myoprocta sp. & - & - & CT & 1 \\
\hline Coendou prehensilis (Linnaeus, 1758) & $\mathrm{LC}$ & - & $\mathrm{O}, \mathrm{E}$ & 1 \\
\hline Mesomys hispidus (Desmarest, 1817) & $\mathrm{LC}$ & - & $\mathrm{M}$ & \\
\hline Proechimys quadruplicatus Hershkovitz, 1948 & $\mathrm{LC}$ & - & TS & \\
\hline Proechimys sp. & - & - & TS & \\
\hline
\end{tabular}

A (1) Montenegro \% Restrepo (2018), (2) Rodríguez-Castellanos et al. (2013), (3) Agudelo-Liz et al. (2018). ICN: Colección de mamíferos "Alberto Cadena García”, Instituto de Ciencias Naturales, Universidad Nacional de Colombia. 
Anexo 2. Números de colección de los especímenes de mamíferos registrados en San José del Guaviare, Colombia incluyendo los especímenes recolectados por nosotros y la revisión de la Colección de Mamíferos del ICN (señalados con *).

Didelphimorphia. Didelphis marsupialis ICN 22576, ICN 22669; Philander andersoni: ICN 22663; Marmosa demerarae: ICN 22542; Marmosa waterhousei: ICN 23829. Pilosa. Choloepus didactylus: ICN 22366. Primates. Cebus albifrons: ICN 22367-72; Alouatta seniculus: ICN 22373, 22654; Lagothrix lagothricha: ICN 22374. Chiroptera. Cormura brevirostris: ICN 22324, 22606-07; Peropteryx macrotis: ICN 22318-19, 22325, 22375, 22382-84, 22400, 22409, 22467, 22488-95, 23830-33; Rhynchonycteris naso: ICN 22546, 22640-41; Saccopteryx bilineata: ICN 22333, 22401; Saccopteryx canescens: ICN 22452; Saccopteryx leptura: ICN 22453, 22567, 22608; Micronycteris hirsute: ICN 23867; Micronycteris megalotis: 22473, 22485, 22674, 23839-40, 23868-71; Lampronycteris brachyotis: ICN 22614, 23838, 23866; Desmodus rotundus: ICN 22563, 23862; Lonchorhina aurita: 22471; Lonchorhina marinkellei: ICN 22472, 23138; Lonchorhina orinocensis: ICN 22308, 22336, 22351-52, 22358-60, 22533, 22568 $69,22589,22591,22599 ;$ Gardnerycteris crenulatum: ICN 22535, 23872-73; Lophostoma brasiliense: ICN 22534; Lophostoma silvicolum: ICN 22414; Tonatia maresi: ICN 23842; Phylloderma stenops: ICN 23874; Phyllostomus discolor: ICN 22592, 22628; Phyllostomus elongatus: ICN 22593, 23841; Phyllostomus hastatus: ICN 22327, 22376 79, 22389-93, ICN 22415, ICN 22474, 22615, 22661; Phyllostomus latifolius: ICN 22309, 22337-39, 22403-04, 22416, 23875-76; Trachops cirrhosus: ICN 22310, 22328, 22417-19, 22427-29, 22486, 22508, 22536, 22600, 23843; Glossophaga commissarisi: ICN 22667; Glossophaga soricina: ICN 22349-50, 22402, 22450, 22458, 22504-07, 22597, 22660; Anoura geoffroyi: ICN 22307, 22457, 22470, 2250203, 22673; Lichonycteris degener: ICN 16191*; Lionycteris spurrelli: ICN 22335, 22598; Hsunycteris thomasi: ICN 23861; Carollia brevicauda: ICN 22304, 22320, 22326, 22410-12, 22423-24, 22440-45, 22454-56, 22468, 2248081, 22496-97, 22501, 22515-19, 22547-49, 22577-82, 22623, 22642-46, 22483, 22447, 22525, 22834, 22878-80;
Carollia castanea: ICN 22520-22554-57, 22583-84, 22609 10, 22646, 23835; Carollia perspicillata: ICN 22305-06, 22321-23, 22334, 22344-48, 22354-57; 22385-88; 22413, 22425-26, 22448-49, 22469, 22482, 22484, 22498-00; 2252124, 22526-32, 22558-62, 22585-88, 22611-13, 22624-27, 22647, 22658-59, 22665-66, 22671-72, 23836-37, 2388183; Glyphonycteris daviesi: ICN 23863; Glyphonycteris sylvestris: ICN 23864; Trinycteris nicefori: ICN 22311, 22420, 22594, 23877; Artibeus lituratus: ICN 22421, 2253738, 22550, 22629; Artibeus obscurus: ICN 22312-13, 2243435, 22509-10, 22590, 22648, 23848; Artibeus planirostris: ICN 22329-31, 22340, 22380-81, 22394-99, 22405; 2243033, 22475, 22511-14, 22539, 22551-52, 22570, 22616-19, 22630, 22649-51, 22662, 22668; Dermanura anderseni: ICN 22564; Dermanura gnoma: 22314-15, 22341, 22422, 22436-38, 23844-47, 23884; Sturnira giannae: ICN 23852; Sturnira tildae: ICN 22359, 22406, 22478, 22566, 2388688; Uroderma bilobatum: ICN 22573, 23854; Uroderma magnirostrum: ICN 22487, 22574; Vampyressa thyone: ICN 23855-56; Mesophylla macconnelli: 22476-77, 22540, 22571, 23849; Platyrrhinus brachycephalus: ICN 22553; 22565, 22575, 22595, 22631-33; Platyrrhinus helleri: ICN 22572; Platyrrhinus infuscus: ICN 22342, 22353, 22652, 2285051, 23885; Eumops auripendulus: ICN 16198; Molossus molossus: ICN 2203*, 22634; Molossus rufus: ICN 2236065, 22407-08; Eptesicus brasiliensis: ICN 22462; Eptesicus chiriquinus: ICN 22461, 22463-65, 22620, 23857; Myotis keaysi: ICN 23889; Myotis riparius: ICN 2237, 22343, 22439, 22466, 22596, 23858, 23890-91; Rhogeessa io: ICN 22451, 22479. Carnivora. Leopardus pardalis: ICN 22541. Artiodactyla. Tayassu pecari: ICN 22892-93; Rodentia. Hyalaeamys sp.: ICN 22605, 22637, 22653, 22655; Neacomys spinosus: ICN 22544, 22545, 22656, Oecomys bicolor: ICN 22638-39, 22657, 22664; Rattus rattus: ICN 16200*, 2260104; Rattus norvegicus: ICN 16199*; Mesomys hispidus: ICN 23859; Proechimys quadruplicatus: ICN 22332, 22621-22, 22635-36, 22670; Proechimys sp.: ICN 22302. 
Hugo Fernando López Arévalo

Universidad Nacional de Colombia.

Bogotá, Colombia

https: / / orcid.org/0000-0002-6692-4308

hflopeza@unal.edu.co

Autor para correspondencia

\section{Darwin Manuel Morales-Martínez}

Universidad Nacional de Colombia.

Bogotá, Colombia

https:/ / orcid.org/0000-0001-5786-4107

dmmoralesm@unal.edu.co

\section{Catherine Mora-Beltrán}

Universidad Nacional de Colombia.

Bogotá, Colombia

https:/ / orcid.org/0000-0002-7294-3716

cmorab@unal.edu.co

\section{María C. Calderón-Capote}

Universidad Nacional de Colombia.

Bogotá, Colombia

https:/ / orcid.org/0000-0002-7646-3082

mccalderonc@unal.edu.co

\section{Catalina Cárdenas-González}

Universidad Nacional de Colombia.

Bogotá, Colombia.

https:/ / orcid.org/0000-0002-5504-5295

ccardenasgo@unal.edu.co

\section{Natalia Atuesta-Dimian}

Instituto Amazónico de Investigaciones Científicas-SINCHI.

Bogotá, Colombia.

https:/ / orcid.org/0000-0003-0845-5168

natuesta@sinchi.org.co

\section{Marco J. Melo}

Cooperativa Ecoturística Playa Güio (COOEPLAG).

Guaviare, Colombia.

https:/ / orcid.org/0000-0003-3108-5628

mjmelo@gmail.com

\author{
Wilmer Ramírez \\ https:/ / orcid.org/0000-0002-3976-250X \\ waramirez69@hotmail.com
}

\section{Mamíferos (Mammalia) de San José del Guaviare,} Colombia

Citación del artículo: López-Arévalo, H.F., Morales-Martínez, D.M., Mora-Neltrán, C., Calderón-Capote, M.C., Cárdenas-González, C., Atuesta-Dimian, N., Melo, M.J. \& Ramírez, R. (2021). Mamíferos (Mammalia) de San José del Guaviare, Colombia. Biota Colombiana, 22(2), 127-146.

https:/ / doi.org/10.21068/c2021.v22n02a07

Recibido: 8 de octubre 2019

Aceptado: 28 de Julio 2020 